Acta Cryst. (1996). C52, 265-278

\title{
International Union of Crystallography \\ Acta Crystallographica Section C Notes for Authors
}

\section{Introduction}

These Notes for Authors provide detailed information on the submission and publication procedures of Acta Crystallographica Section C: Crystal Structure Communications. They embody the latest policy of the IUCr Commission on Journals on the rapid publication of crystal structure studies and supersede those of Acta Cryst. (1995). C51, 159-174.

Authors are expected to submit Section $C$ papers in Crystallographic Information File (CIF) format. Hard-copy submissions of manuscripts will not normally be accepted.

The Notes are divided into the following sections:

$\S 2$. Publication requirements

§3. Published material

$\S 4$. Supplementary material

$\S 5$. Submission procedure

$\S 6$. Diagrams

\$7. Nomenclature

§. References

§. File transfer

$\S 10$. Electronic status information

\$11. Reprints

Appendix 1. Guidelines for editing CIF text Appendix 2. Essential items for the Experimental section Appendix 3. Transfer of Copyright Agreement form Appendix 4. Annotated example of a Section $C$ paper

\section{Publication requirements}

\subsection{Address for submissions}

All papers submitted to Section $C$ should be sent to the $\mathrm{IUCr}$ Offices in Chester.

Publication material submitted electronically in CIF format on magnetic media, or as hard-copy (diagrams and Transfer of Copyright Agreement form), should be sent by airmail or courier to:

\section{The Managing Editor \\ International Union of Crystallography \\ 5 Abbey Square \\ Chester CH1 2HU \\ England}

Telephone: 441244342878

Fax: $\quad 441244314888$

E-mailed manuscripts and data in CIF format should be sent to:

\section{med@iucr.ac.uk}

or, for large data files, transmitted by ftp (see §9) to:

ftp.iucr.ac.uk (192.70.242.1)

\subsection{Languages of submission}

The languages of publication are English, French, German and Russian.

\subsection{Author's warranty}

The submission of a paper is taken as an implicit guarantee that the work is original, that it is the author(s) own work, that all authors concur with and are aware of the submission, that proper credit is given to others, that the manuscript has not been published (in any language), and that it is not being considered and will not be offered elsewhere while under consideration for an IUCr journal. For these reasons, the submission must be made over the signature of at least one author.

\subsection{Copyright}

Except as required otherwise by national laws, an author must sign and submit a copy of the Transfer of Copyright Agreement form (Appendix 3 ) for each manuscript before it can be accepted.

\subsection{Categories of submission}

Section $C$ publishes two categories of papers:

(i) The primary purpose of Section $C$ is to publish papers that report the results of one or more crystal structure determinations. The format of these papers is described in $\S 3$. Submissions which exceed these guidelines will, at the discretion of the Co-editor and referees, be shortened for publication in Section $C$, or be transferred to Section $B$.

(ii) Section $C$ also publishes Addenda and Errata to previously published papers. These are used for reporting additions to, comments on, or errata in existing Section $C$ publications and are not intended for interim reports of work in progress. These manuscripts should not exceed 1000 words and, while being refereed as for (i), will be processed as rapidly as possible.

\subsection{Method of submission}

Manuscripts should be submitted to the Chester office electronically in CIF format. E-mail or magnetic media submission is accepted.

\subsection{Handling of manuscripts}

A paper submitted to Chester will be first checked for completeness (note the availability of a CIF pre-checking facility discussed in \$5.1). If the material is incomplete or inadequate it will be returned to the author for correction. Numerical data are then checked for internal consistency, correct symmetry and cell dimensions, and sensible structural geometry. Checks will also be made for previous publication of the structure(s) contained in the paper. The results of these checks are summarized in a Check Report.

The paper, together with the Check Report, is forwarded to a Co-editor, who is in charge of the reviewing steps and future communications with the authors, up to the acceptance stage.

If a paper passes the checking process without significant fault it may be designated as a fast-track submission and sent, with a full advance proof, to a Co-editor for rapid reviewing. If the paper is accepted without significant change, the advance proof will be forwarded to the author for final approval. The fast tracking of papers offers significantly shorter publication times to authors who submit well prepared and carefully checked 
manuscripts. This service is possible because of the reductions in workload that such submissions afford the editorial staff.

If changes to a manuscript requested by the editorial staff or the Co-editor are not received within six months of transmittal to the author, the submission will automatically be withdrawn. Any subsequent communication of the material will be treated as a new submission in the editorial process.

Once a paper is accepted, it is the responsibility of the Managing Editor to prepare the paper for printing and to correspond with the authors and/or the Co-editor to resolve publication ambiguities or inadequacies. The date of acceptance that will appear on the published paper will be the date on which the Managing Editor receives the last item needed.

\subsection{Selection of Co-editor}

Co-editors are assigned to a paper according to their current workload and expertise. Authors may indicate a preference for a particular Co-editor but the editorial staff reserve the right to make the final choice.

\subsection{Author grievance procedure}

An author who believes that a paper has been unjustifiably rejected by the Co-editor may appeal initially to the Section Editor, and then to the Editor-in-Chief if still aggrieved by the decision.

\section{Published material}

The following material is required for publication in Section $C$. Details of these categories are given in $\$ 4$. An example of a published paper is shown in Appendix 4.

\subsection{Title and authors}

The Title should be both short and informative. Avoid complicated IUPAC names and redundant phrases such as 'The Structure of ...' or 'Crystal Structure of ...' should be removed. It is preferred that the full first name of each author be used. The contact author should, where possible, provide an e-mail address. This will be used for editorial communications and will normally appear in the published paper.

\subsection{Abstract}

The Abstract must be written in English and should summarize only the most important aspects of the study. It should not contain the crystal data. The systematic IUPAC name and the chemical formula should be given here, if they are not included in the Title.

\subsection{Comment}

The Comment section should contain:

(i) Concise reasons why the structure determination was undertaken.

(ii) The origin of the material studied. General chemical background material and references should be included. Details of the chemical extraction, synthesis and crystallization processes should be given in the Experimental section (see \$3.6).

(iii) Any novel and unusual features of the coordination, bonding, geometry, conformation, crystal packing, etc. Avoid discussions of geometry data which agree with established values (see Volume C of International Tables for Crystallography, pp. 681-791).

\subsection{Acknowledgements}

\subsection{References}

References to published work must be cited in the format detailed in $\S 8$. If reference is made to unpublished work, prior consent must be first obtained from the authors.

\subsection{Experimental}

Experimental details will be tabulated under the subheadings Crystal data, Data collection and Refinement. Any discussion of experimental procedure is to be limited to novel or unusual features and should be given in grammatically correct text.

A list of experimental data requested is provided in Appendix 2. These represent the primary information about the structure. If they are incomplete or inconsistent the manuscript will be returned to the authors as being inadequate. Authors should take particular note of the following editorial acceptance criteria.

(i) The chemical formula, chemical formula weight, volume of unit cell, space group and formula units per cell, $Z$, must be consistent with the atomic sites (see $\$ \$ 3.7$ and 4.1).

(ii) The cell and space group must encompass the highest symmetry permitted by the diffraction intensities. Special care is needed if $Z$ exceeds the number of general equivalent positions for a space group, e.g. check if multiple diffraction has affected the systematically absent intensities.

(iii) The precision of a structure is determined largely by the number and the reliability of the diffraction measurements. Unless there are mitigating circumstances, diffraction data should extend to at least $\sin \theta / \lambda=0.6 \AA^{-1}$ (i.e. $\theta_{\max } \geq 25^{\circ}$ for Mo $K \alpha$; $\theta_{\max } \geq 68^{\circ}$ for $\mathrm{Cu} K \alpha$ ).

(iv) Absorption corrections must be appropriate to the crystal and the precision of the analysis. If $\mu$ is less than $0.3 \mathrm{~mm}^{-1}$ and the crystal is regular, absorption corrections are usually unnecessary. Refined absorption techniques (based on $\Delta F$ ) should be employed only if analytical or $\psi$-scan methods cannot be applied. The calculated maximum and minimum transmission values should be physically sensible with respect to the material and the crystal shape.

(v) An $R$ value calculated with partial reflection data is not a good measure of structure reliability. The precision of a structure is better gauged from the estimated standard deviations of the parameters. These e.s.d.'s are critically dependent on the number and the reliability of the measured structure factors used in the refinement process. Usually, a low 'observed' threshold (see items 20 and 21 in Appendix 2) means that more reflections are used in the refinement (see item 30). This is more important to structure reliability than raising this threshold in order to lower the $R$ value. Provided that the least-squares weights incorporate the e.s.d.'s of the measured intensities, low thresholds of say $\sigma(I)$ or $2 \sigma(F)$ are recommended. For most analyses there are advantages in using all data in the least-squares refinement.

\subsection{Table of atomic sites}

A table containing atomic site data will be published. This will include the e.s.d.'s of the coordinates and displacement parameters. Information about hydrogen-atom sites will be printed only at the Co-editor's discretion. (See $\$ \S 5.5$ for submission details.)

\subsection{Table of selected geometrical data}

A table of the bond distances, interatomic contacts, bond angles and torsion angles of special importance to the structure and relevant to the discussion in the Comment section will be published. All other geometrical data will be archived. The data to be published may be amended by the Co-editor. (See $\$ 5.6$ for submission details.) 


\subsection{Chemical structural diagram}

Papers reporting the structure of a molecular compound must contain a chemical structural diagram (see $\$ 6$ and the example in Appendix 4).

\subsection{Crystallographic diagram(s)}

Papers should contain one crystallographic diagram of each structure. For papers reporting molecular structures this should be a molecular diagram; otherwise it should be a packing or polyhedron diagram. Unique atom sites should be identified with labels as specified in \$7.1. The atom labels used in the diagram should be consistent with those in the table of atom coordinates (83.7). Additional diagrams are at the Co-editor's discretion.

The importance of high quality diagrams is detailed in $\S 6$. A clear, well presented crystallographic diagram is an essential component of the published material. It encapsulates information about the stereochemistry, the geometry, and, if it is a displacement ellipsoid plot, about the structural disorder and thermal motion. A displacement ellipsoid diagram is required, either for publication or as supplementary material to be used in the review process.

\subsection{Contents requirements}

The Table of Contents of the journal will list each paper with the title and author(s), a chemical structural diagram for molecular compounds, and a written synopsis for compounds which cannot be shown as a chemical structural diagram. A synopsis should be one or two sentences (less than 40 words) in length.

\section{Supplementary material}

Supplementary material is required for reviewing and checking the submission. This material will be archived but is not usually published. The following categories of data are requested.

\subsection{Hydrogen-atom sites}

Hydrogen-atom sites may, at the Co-editor's discretion, be published in the paper.

\subsection{Anisotropic displacement parameters}

Anisotropic displacement parameters (preferably as $U_{i j}$ 's) may, at the Co-editor's discretion, be published in the paper. If parameters other than $U_{i j}$ 's are used, the exact form of the temperature factor expression must also be indicated.

\subsection{Geometrical data}

A list of the important interatomic bond lengths and intermolecular non-bonded contact distances must be provided for checking purposes. Valence and torsion angles may be included if desired. Selected intermolecular distances and torsion angles may, at the Co-editor's discretion, be published in the paper.

\subsection{Reflection data}

A list of the reflection data $h, k, l, Y_{\text {meas }}, Y_{\text {calc }}, \sigma Y_{\text {meas }}$ (where $Y$ is $F, F^{2}$, or $I$ ), must be submitted. Where possible, this should be supplied as a single electronic file in CIF format (see $\$ \$ 5.7$ and 9), otherwise as a hard-copy listing. Authors should indicate if the $Y$ values have been corrected for absorption and extinction effects.

\subsection{Miscellaneous data}

Miscellaneous data (including diagrams) may be deposited at the discretion of the Co-editor. If the submitted crystallographic diagram (see §3.10) does not show the displacement-parameter ellipsoids, such a diagram is required for the checking and reviewing process.

Details of the parameter constraints and restraints applied to the refinement process must be supplied as supplementary material unless they are described in the experimental refinement procedure (see item 40 in Appendix 2).

\subsection{Powder diffraction data}

For papers that present results based on powder diffraction data, the numerical intensity of each measured point on the profile (as a function of scattering angle) will be deposited with the IUCr and sent by the Co-editor to the International Center for Diffraction Data (ICDD), 12 Campus Boulevard, Newton Square, PA 19073-3273, USA. These data will be checked and assigned an ICDD reference number which will, where possible, be published in the paper. Papers reporting Rietveld refinements should include a figure showing the diffraction profile and the difference between the measured and calculated profiles.

\section{Submission procedure}

\subsection{General information}

Authors are required to submit papers electronically in CIF format. The required data items are described briefly below and in Appendix 2. Detailed descriptions of these items are given in the CIF Core Dictionary [Hall, Allen \& Brown (1991). Acta Cryst. A47, 655-685]. The special requirements for text items in a CIF are summarized in Appendix 1, and an example of a published paper is given in Appendix 4. Authors are also referred to the Guide to CIF for Authors, which is available free of charge from the Editorial Office.

It is anticipated that the bulk of the numerical data in a CIF will be generated by standard software, whereas the fields containing text for the sections Abstract, Comment, etc. (see \$5.3) will be added by the author using a text editor.

The data items listed below, and summarized in Appendix 2, will be extracted automatically by the CIF-handling software in Chester. Authors who wish to include data items not listed below should identify these in the contact letter field (see \$5.2). Otherwise these data items will be ignored by the extraction process. (The present CIF Core Dictionary contains only data names for conventional single-crystal structure studies. Data names for powder diffraction and protein structure items are available from the Managing Editor.)

Note that a CIF may also be used to transmit supplementary publication information. Authors are advised, however, not to transmit reflection data or diagrams as part of the standard $\mathrm{CIF}$, as the size of these items may cause e-mail problems or exceed the capacity of some diskettes. Nevertheless, authors are requested, whenever possible, to submit these data electronically using the procedures described in $\$ \$ 5.7$ and 9 .

The submission of a manuscript will normally be in two parts: (i) a machine-readable file or files containing data items from $\S \S 3$ and 4 and (ii) a signed Transfer of Copyright Agreement form accompanied by the balance of items as hard-copy material.

A CIF pre-checking facility is provided by the Editorial Office. Authors are encouraged to check a CIF, prior to submission, by sending it, without any attachments, such as the sender's signature script, to the e-mail address

\section{checkcif@iucr.ac.uk}

Tests will be made automatically for file integrity, mandatory data and sensible cell, symmetry and geometry information, and a Check Report will be returned to the sender's e-mail address. The tests are less extensive than those applied during 
checking of a paper submitted for publication, but pre-checking will significantly reduce the delays which occur with incorrect or inadequate CIF submissions.

The Editorial Office also provides a manuscript previewing facility for authors. Following an error-free Check Report from checkcif (see above), authors are encouraged to e-mail the same CIF to

\section{printcif@iucr.ac.uk}

which will return a PostScript file of the paper in the form that is sent to the Co-editors for reviewing. This file can be printed locally so that authors can make final checks on the text and tables, prior to submission.

On submission of the CIF for publication, the numerical data, diagrams and text are checked for completeness and a printed copy of the paper is forwarded to a selected Co-editor for use in the review process. Any amendments to the paper during this review must be indicated on the printed manuscript, as this document is used by the editorial staff to update and revise the archived CIF. An author-modified CIF should not be resubmitted except at the explicit request of the Co-editor. Such a request will be made only if there are major changes to the data (e.g. re-refinement of the structure). In such cases the resubmitted CIF should be sent to Chester and must contain a publ_contact_letter field stating the Co-editor's manuscript reference cōe.

\subsection{Initial publication request}

Details of the contact author and requested journal should be entered into the submitted CIF as the data items:

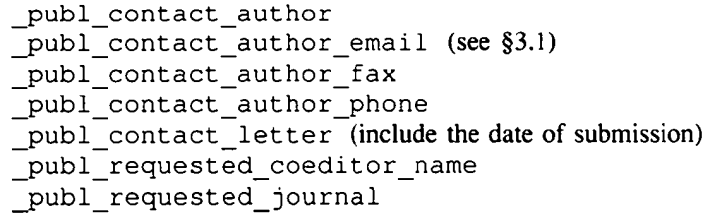

\subsection{Manuscript text}

The manuscript text information is entered as the data items:

$$
\begin{aligned}
& \text { _publ_author_name } \\
& \text { _publ_author_address } \\
& \text { _publ_section_title } \\
& \text { _publ_section_abstract } \\
& \text { _publ_section_comment } \\
& \text { _publ_section_expt l_prep (see } \$ 5.4 \text { for use) } \\
& \text { _publ_section_exptl_refinement (see } \$ 5.4 \text { for use) } \\
& \text { _publ_section_experimental (see } \$ 5.4 \text { for use) } \\
& \text { _publ_section_acknowledgements } \\
& \text { _publ_section_references } \\
& \text { _publ_section_figure_captions } \\
& \text { _publ_section_table_legends }
\end{aligned}
$$

These text items are entered with a standard ASCII text editor. Guidelines for entering text are given in Appendix 1.

\subsection{Experimental data}

The experimental data should be submitted as the specific data items listed in Appendix 2. Most of these items are numerical values (with the estimated standard deviations appended in parentheses, if applicable) but some are descriptive text. Several require the use of standard codes from the CIF Core Dictionary; these items are flagged with a $\dagger$ in Appendix 2.

The two new data items in this section_publ_section exptl_prep and_publ_section_exptl_refinement are intended for detailed descriptive text. The former should give sufficient information on the chemical and crystal preparation and identification (e.g. on melting points and densities), to reproduce the experiment, and the latter should detail any special aspects of the structure determination and refinement. The older _publ_section_experimental field may be used if the experimental discussion cannot be categorized in this way.

\subsection{Atomic site data}

Atomic site information is entered as a list of data items. The following items must be supplied:

$$
\begin{aligned}
& \text {-atom_site_label } \\
& \text {-atom_site_fract_x } \\
& \text {-atom_site_fract_y } \\
& \text {-atom_site_fract_z } \\
& \text {-atom_site_U_iso_or_equiv }
\end{aligned}
$$

Additional items can be included where appropriate e.g. site occupancies as _atom_site_occupancy or disorder flags as _atom_site_disorder_group. The rules for labelling atom sites are detailed in $\$ 7 . \overline{1}$.

\subsection{Geometrical data}

Structural geometry data (distances and angles) are entered as separate lists, in the data item categories:

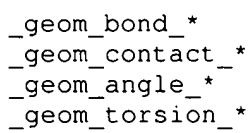

The data item *_publ associated with each distance or angle value that is to be published in a table must be set as yes.

\subsection{Supplementary material}

Supplementary data, as specified in $\$ 4$, are needed for the checking and reviewing process. Reflection data and diagrams (as HPGL or PostScript files) can be sent as a separate e-mail, or transferred using the ftp procedures described in $\$ 9$

\subsection{Non-CIF material}

A signed Transfer of Copyright Agreement form must be sent to Chester by post or fax. If diagrams and supplementary data cannot be transmitted by ftp (see $\$ 9$ ) the following items should to be sent as hard-copy by post or courier:

(i) a chemical structural diagram, in triplicate (see \$3.9);

(ii) a crystallographic diagram, in triplicate (see $\$ \$ 3.10$ and 6);

(iii) a single listing of reflection data (see $\$ 4.4$ );

(iv) other supplementary material, in triplicate (see \$6.4).

\subsection{CIF submission by Internet}

A CIF submission by network transmission should be directed to the Managing Editor's e-mail address (see \$2.1). Receipt will be acknowledged by e-mail and a reference number assigned.

Associated hard-copy material (as per \$5.8) should be sent, quoting this reference number, by post or courier to the Managing Editor's postal address (see §2.1).

\subsection{CIF submission by magnetic media}

A CIF submission by diskette or magnetic tape should be sent, along with the associated hard-copy material (see §5.8), to the Managing Editor's postal address (see \$2.1). Receipt will be acknowledged. The following media formats may be used:

3.5 in IBM-compatible diskettes.

5.25 in IBM-compatible diskettes.

3.5 in Apple Macintosh diskettes.

3.5 in Sun OS diskettes (cpio format).

0.5 in $1600 / 6250$ bpi magnetic tape. 


\section{Diagrams}

\subsection{Design}

The choice of figures, and the associated tables, should be optimized to produce the shortest possible printed paper consistent with clarity. Duplicate presentation of the same information in tables and figures is to be avoided, as is redundancy within the text. Distances and angles should be given as a table rather than displayed in the crystallographic diagram.

A chemical structural diagram must be included for molecular compounds (see Appendix 4 for a typical example).

\subsection{Quality}

It is essential that diagrams be of publication quality (see $\S \S 3.9$ and 3.10). If diagrams are submitted as hard copy, rather than electronically (see $\$ \S 5.8$ and 9), they must be high-resolution black and white originals or very good photocopies. Submissions containing diagrams of poor quality will not be processed until this is rectified.

\subsection{Colour figures}

Figures in colour are accepted at no cost to the author provided that the Editor agrees that they improve the understanding of the paper. They should be provided as glossy prints or slides; laser printer or photocopier output will generally be unsatisfactory for colour reproduction. Slides should be accompanied by a photocopy showing the required figure layout.

\subsection{Size}

Each diagram should be provided on a separate sheet of about A4 size $(210 \times 297 \mathrm{~mm})$. These will normally be reduced by the printer to fit one-column width $(80 \mathrm{~mm})$. Authors are requested not to make this reduction, but should take care that the orientation and labelling of the diagram is optimal for a one-column presentation. Figures at two-column width will be at Co-editorial discretion.

\subsection{Lettering and symbols}

Fine-scale details and lettering must be large enough to be clearly legible (not less than $1.2 \mathrm{~mm}$ in height) when the diagram is one column $(80 \mathrm{~mm})$ in width. Lettering should be kept to a minimum. Atom site labels in crystallographic diagrams should match labels used in the atom site lists and text. Distance, bond angle and torsion angle values should be given as tables and not appear in a diagram. Descriptive matter should be placed in the legend. If a packing diagram is submitted it must show clearly the cell axes and orientation.

Author generated lettering is preferred on diagrams and figures. If necessary, lettering can be added by the printer if a photocopy showing the required lettering is supplied with an unlettered original.

\subsection{Numbering and legends}

Diagrams and photographs are to be numbered as figures in a single series, normally in the order in which they are referred to in the text. A list of the legends ('figure captions') should be included in the CIF. Legends of figures containing displacement ellipsoids must state the probability limit used.

\section{Nomenclature}

\subsection{Crystallographic nomenclature}

Atoms of the same chemical species within an asymmetric unit should be identified by unique labels composed of a number appended to the IUPAC chemical symbol (e.g. C5, C7 etc.).
These numbers need not be placed in parentheses. Chemical and crystallographic numbering should be in agreement wherever possible. Crystallographically equivalent atoms in different asymmetric units should be identified with lower-case roman numeral superscripts appended to the original atom labels (e.g. $\mathrm{C}^{\mathrm{i}}$ and $C 7^{\text {iv }}$ ).

Space groups should be designated by the Hermann-Mauguin symbols. Standard cell settings, as listed in Volume A of International Tables for Crystallography, should be used unless objective reasons to the contrary are stated. When a non-standard setting is used, the list of equivalent positions should be given. Hermann-Mauguin symbols should also be used for designating point groups and molecular symmetry. It is helpful if the origin used is stated explicitly where there is a choice. The choice of axes should normally follow the recommendations of the Commission on Crystallographic Data [Kennard, Speakman \& Donnay (1967). Acta Cryst. 22, 445-449].

\subsection{Nomenclature of chemical compounds}

Names of chemical compounds and minerals are sometimes ambiguous. Authors should therefore give the molecular formula and the chemical structure diagram for each molecular compound referred to in the manuscript.

Chemical formulae and nomenclature should conform to the rules of nomenclature established by the International Union of Pure and Applied Chemistry (IUPAC), the International Union of Biochemistry and Molecular Biology (IUBMB), the International Mineralogical Association and other appropriate bodies. As far as possible the crystallographic nomenclature should correspond to systematic usage.

Any accepted trivial or non-systematic name may be retained, but the corresponding systematic (IUPAC) name should also be given. If help on assigning systematic names is sought from advisory sources, authors are requested to indicate the source consulted.

\subsection{Units}

The SI system of units is to be used except that the ångström (symbol $\AA$, defined as $10^{-10} \mathrm{~m}$ ) is preferred to the nanometre $(\mathrm{nm})$ or picometre $(\mathrm{pm})$.

Recommended prefixes of decimal multiples should be used rather than $\times 10^{n}$ '.

\section{References}

References to published work must be indicated by giving the authors' names followed immediately by the year of publication, e.g. Neder, Frey \& Schulz (1990) or (Neder, Frey \& Schulz, $1990)$. Where there are six or more authors the reference in the text should be indicated in the form Smith et al. (1989) or (Smith et al., 1989) etc.

In the reference list, entries for journals [abbreviated in the style of Chemical Abstracts (the abbreviations Acta Cryst., J. Appl. Cryst. and J. Synchrotron Rad. are exceptions)], books, multi-author books, computer programs, personal communications and undated documents should be arranged alphabetically and conform with the following style:

Bürgi, H.-B. (1989). Acta Cryst. B45, 383-390.

Hervieu, M. \& Raveau, B. (1983a). Chem. Scr. 22, 117-122.

Hervieu, M. \& Raveau, B. (1983b). Chem. Scr. 22, 123-128.

Hummel, W., Hauser, J. \& Bürgi, H.-B. (1996). In preparation.

Jones, P. T. (1987). Personal communication.

McCrone, W. C. (1965). Physics and Chemistry of the Organic Solid State, Vol. 2, edited by D. Fox, M. M. Labes \& A. Weissberger, pp. 725-767. New York: Interscience. 
Perkins, P. (undated). PhD thesis, University of London, England.

Sheldrick, G. M. (1976). SHELX76. Program for Crystal Structure Determination. University of Cambridge, England.

Smith, J. V. (1988). Chem. Rev. 88, 149-182.

Smith, J. V. \& Bennett, J. M. (1981). Am. Mineral. 66, 777-788.

Vogel, A. (1978). Textbook of Practical Organic Chemistry, 4th ed. London: Longman.

Note that inclusive page numbers must be given.

When more than ten references are taken from a data base (usually for a structural paper), a condensed reference notation of the Coden type should be used.

\section{File transfer}

Authors with computer access to the Internet may use anonymous file transfer protocol (ftp) to transfer large electronic files to the Editorial Office in Chester. Files larger than $70 \mathrm{~K}$ bytes should be transferred in this way, smaller files can be sent by e-mail.

The procedure for transferring files by ftp is described below. Files need to be deposited in a directory called incoming' with a filename constructed from the reference number supplied by Chester on receipt of the initial CIF submission. Files containing refiection data in CIF format should be identi- fied by the filename extension .hkl. Files containing diagrams in HPGL or PostScript format should be given the extensions .hpg or .ps, respectively. Multiple files for the same submission should be identified by filenames constructed as ref.id.ext where id indicates the contents, e.g. $x z 1087 . \mathrm{figl} . \mathrm{ps}$ and xz1087.fig2.ps.

The procedure for transferring files is shown in Fig. 1.

\section{Electronic status information}

Authors may obtain information about the current status of their papers via the world-wide web at the address http://www.iucr.ac.uk/docs/status.html/ (authors will need to provide the Co-editor reference number of their paper and the last name of one of the authors) or by e-mail by sending an email message to status@iucr.ac.uk with the Co-editor reference number and the name of one of the authors as the subject line (e.g. JA0325 Smith). The body of the message should be empty. A status report will be returned by e-mail.

\section{Reprints}

Twenty-five reprints of each published article will be provided to the correspondence author free of charge. (i) On your workstation enter:

(ii) Wait for Name ...: prompt and enter:

(iii) Wait for Password: prompt and enter:

(iv) Wait for $\mathrm{ftp}>$ prompt and enter:

(v) Transfer a file from your account (e.g. b28.cif) as an identifiable name (e.g. zb1032.hki):

(vi) Wait for ftp> prompt before sending another file

(vii) Finish off the ftp session by entering:

(viii) Send an e-mail to Chester (medeiucr.ac.uk) with a list of the files transferred by ftp

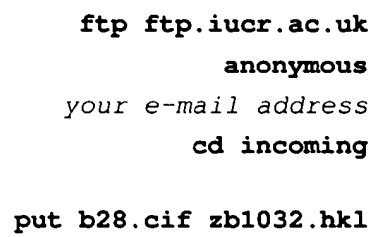

bye

Fig. 1. Procedure for transferring files. 
APPENDIX 1

Entry guidelines for CIF text

The text data items in a CIF must be entered as standard ASCII printable characters (those with decimal integer values of 32 to 126). Non-printing characters should not be used. No attempt should be made to indicate italic or bold characters.

Superscript characters should be bounded by the symbol " "; e.g. enter $\mathrm{C}^{\wedge} \mathrm{ii}{ }^{\wedge}$ for $\mathrm{C} 5^{\mathrm{ii}}, 10^{\wedge}-2^{\wedge}$ for $10^{-2}$.

Subscript characters should be bounded by the symbol " "; e.g. enter $\mathrm{U}^{\sim} 12^{\sim}$ for $U_{12}$.

Greek characters may be entered as digraphs in which the backslash character ' $Y$ ' is used to signal that the next character should be treated as a special symbol.

la $\alpha \backslash \mathrm{b} \beta \backslash \mathrm{g} \gamma \backslash \mathrm{d} \delta \backslash \mathrm{e} \epsilon \backslash \mathrm{z} \zeta$ h $\eta \backslash \mathrm{q} \theta$ i $\iota \mathrm{k} \kappa \vee \lambda \backslash \mathrm{m} \mu$

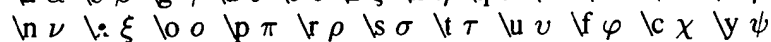

Iw $\omega \backslash A$ A $\backslash B$ B \G $\Gamma$ etc.

Accented characters are represented in a similar way to Greek characters:

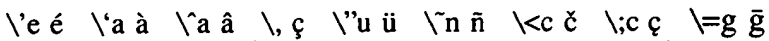

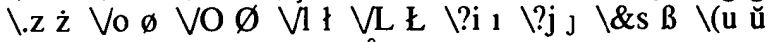
\> ô $1 \%^{\circ} 1 \%$ a å $1 \%$ A $\AA$

If other accents need to be indicated for characters used in CIF text, supply these as a separate hard-copy sheet.

Note that special characters will normally only be used in publ_data items. Do not use them in _chemical_formula_ or_symmetry_space_group_name_data items. 
APPENDIX 2

CIF data items included in the Experimental section

The following data should be submitted as discrete CIF data items and not as text items, with the exception of the data items publ_section_expt1_prep and_publ_section_expt__refinement (see §5.3). The items flagged with a $\dagger$ should be supplied as one of the standard codes listed in the CIF Core Dictionary.

\section{Crystal data}

CIF data names

1. Chemical and crystal source or preparation (see $§ 5.3$ )

publ_section_exptl_prep

2. Chemical formula

chemical_formula_sum
_chemical_formula_moiety

3. Chemical formula weight $M_{r}$ (Da)

_chemical_formula_weight

4. Cell setting $\dagger$

5. Space group

6. Unit-cell dimensions $\left(\AA,{ }^{\circ}\right)$

7. Volume of unit cell $V\left(\AA^{3}\right)$

8. Formula units per cell $Z$

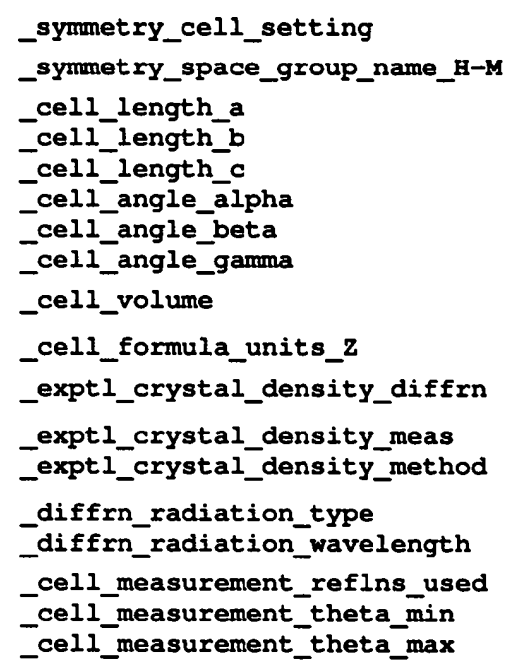

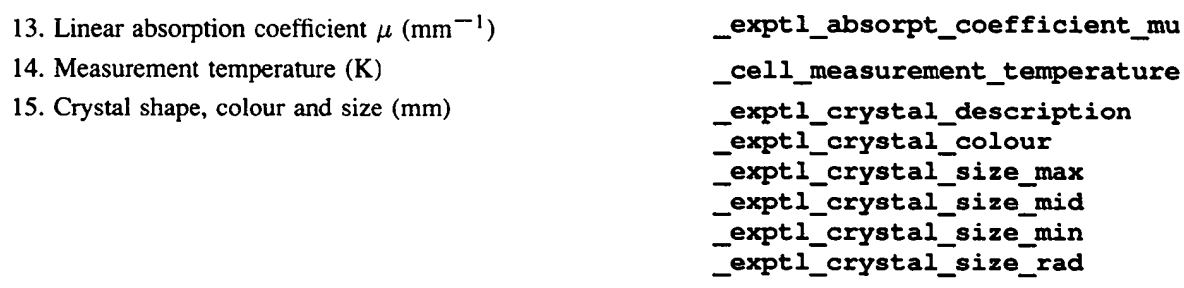

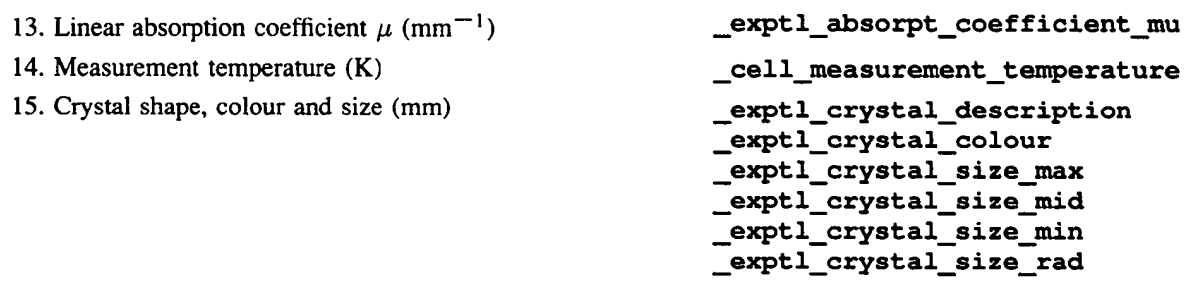

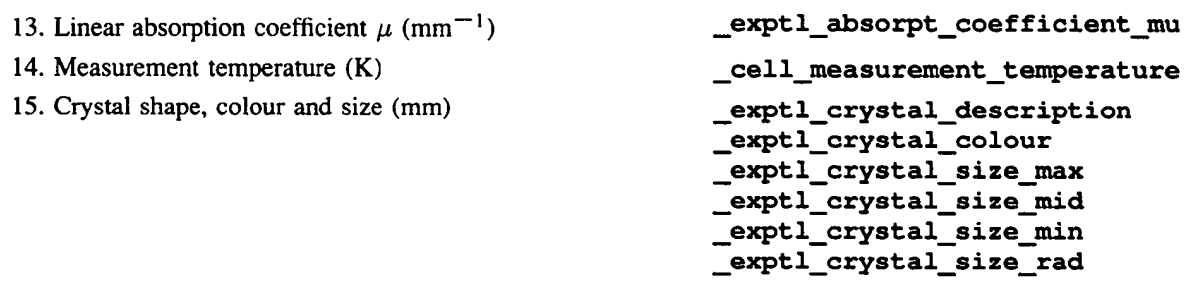

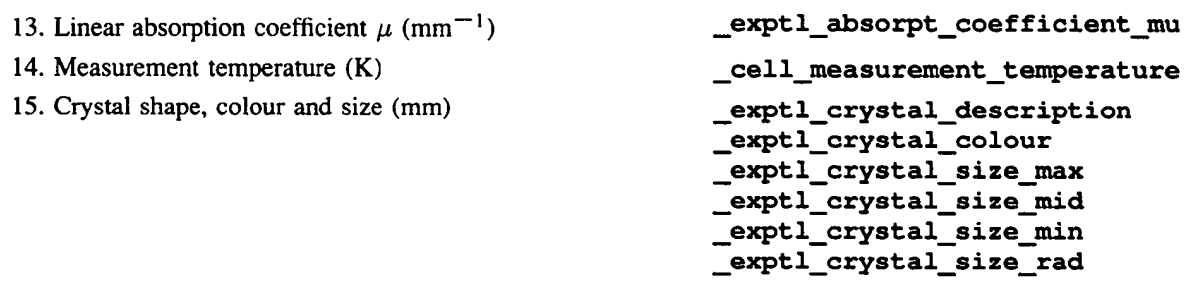

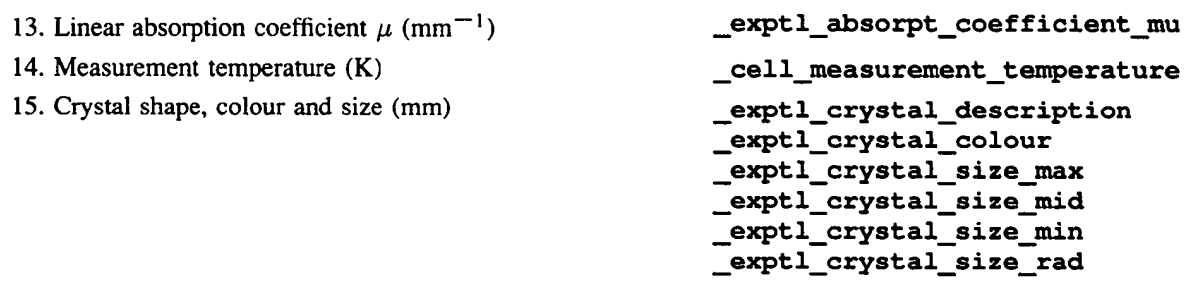

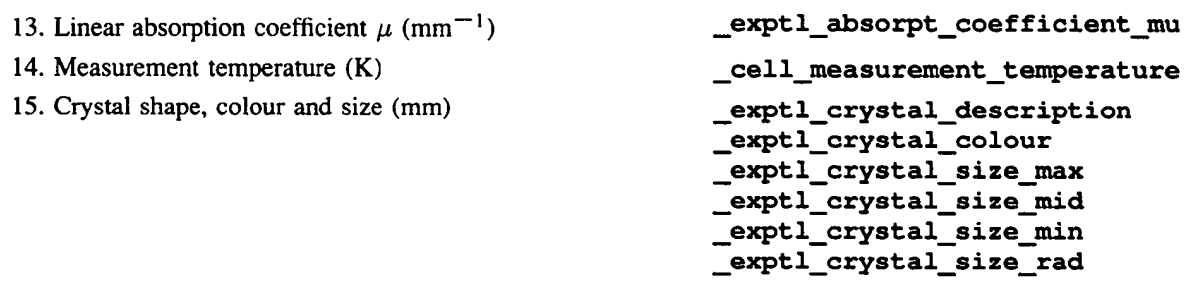

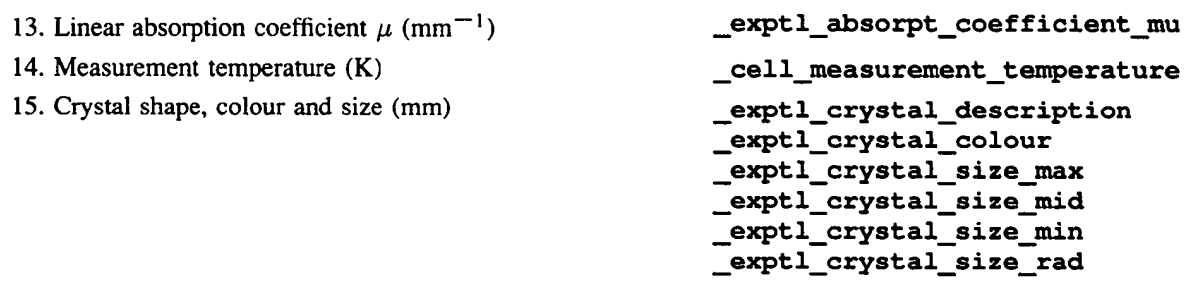

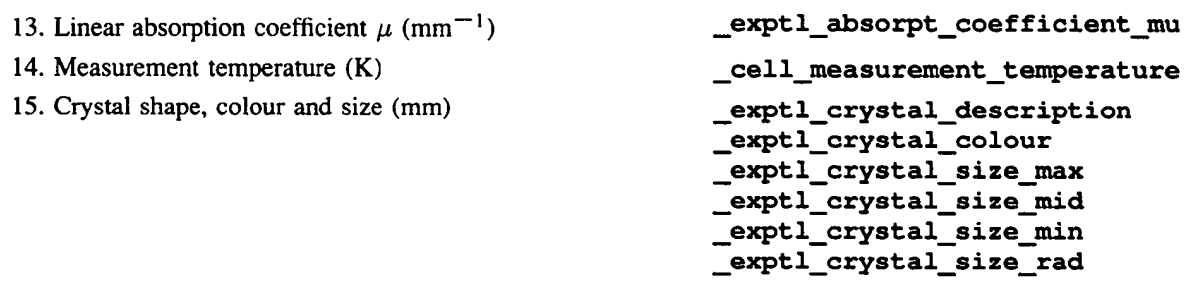




\section{APPENDIX 3 \\ International Union of Crystallography \\ Transfer of Copyright Agreement}

Title of Article (Please type or use capital letters)

Authors (Please type or use capital letters)

Copyright to the above article is hereby transferred (except as otherwise required by national laws*) to the International Union of Crystallography, effective if and when the article is accepted for publication in Acta Crystallographica, Journal of Applied Crystallography or Journal of Synchrotron Radiation. In addition to reproduction in conventional printed form your article will be stored electronically. Your assignment of Copyright signifies your agreement to the journal making arrangements to include your paper in document delivery services, CD-ROMs and electronic databases, etc., worldwide. This transfer of copyright also applies to data submitted in machine-readable form. However, the authors reserve the following:

(1) All proprietary rights other than copyright, such as patent rights.

(2) The right to use all or part of this article in future works prepared by or on the behalf of the author.

(3) In the case of 'work for hire', the right of the employer to incorporate all or part of this article in future works.

(4) The royalty-free right of the employer to make copies of this article, without further permission, for his or her own use but not for resale. Any identification or notice appearing on the pages to be reproduced must not be deleted.

Signature

Name and position, if not author

Date

This Transfer of Copyright Agreement must be signed by at least one of the authors (who agrees to inform the others, if any) or, in the case of a 'work made for hire', by the employer.

By signing this form you certify that your contribution is your original work, has not been published before and is not being considered for publication elsewhere; that you have obtained permission for and acknowledged the source of any excerpts from other copyright works; and that to the best of your knowledge your paper contains no statements which are libellous, unlawful or in any way actionable.

The signed statement must be received before the article can be accepted for publication. Requests for further information should be sent to the Executive Secretary of the Union.

* In this case please give further details overleaf.

For use of the International Union of Crystallography only.

\begin{tabular}{|c|c|c|c|}
\hline M. E. Ref. & Co-editor Ref. & Issue & Journal \\
\hline
\end{tabular}

This form may be photocopied. 
APPENDIX 4

Annotated example of a Section $C$ paper

\section{The First Dinuclear Cobalt-Rhenium Cluster Compound: Hexacarbonylbis $(\mu-$ dicyclohexylphosphanido)cobaltrhenium}

\author{
ULRICH FLÖRKE AND HANS-JÜRGEN HAUPT \\ Anorganische und Analytische Chemie, Universität-GH \\ Paderborn, Warburgerstrasse 100, D-4790 Paderborn, \\ Germany.E-mail: floe@mvaxac.uni-paderborn.de
}

(Received 12 March 1993; accepted 18 May 1993)

\section{Abstract}

The title compound [hexacarbonyl- $1 \kappa^{4} C, 2 \kappa^{2} C$-bis $(\mu$ dicyclohexylphosphido- $\left.1: 2 \kappa^{2} \mathrm{P}\right)$ cobaltrhenium $(\mathrm{Co}-\mathrm{Re})$

0.5-methanol solvate, $\left[\mathrm{CoRe}\left(\mathrm{C}_{12} \mathrm{H}_{22} \mathrm{P}\right)_{2}(\mathrm{CO})_{6}\right] .0 .5\left(\mathrm{CH}_{3}\right.$ $\mathrm{OH})$, belongs to a type of edge-linked tetrahedronoctahedron coordination complex. The molecule has local $C_{2 v}$ symmetry with ecliptic conformation of the carbonyl ligands at both metal centres, and a $\mathrm{Co}-\mathrm{Re}$ single-bond length of 2.786 (1) $\AA$.

\section{Comment}

In the course of our work on phosphanido-bridged homoand heteronuclear metal-atom cluster compounds (Haupt, Balsaa \& Flörke, 1988; Haupt, Heinekamp \& Flörke, 1989; Flörke \& Haupt, 1993), we isolated the title compound (I) in which a $\mathrm{Co}-\mathrm{Re}$ bond is symmetrically bridged by two dicyclohexylphosphanido groups.

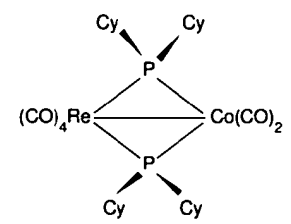

(I)

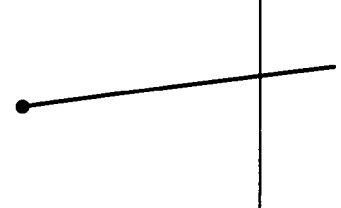

not
The Co atom has distorted tetrahedral coordination from two carbonyl ligands and the bridging $\mathrm{P}$ atoms. These bridging atoms and four of the carbonyl groups give rise to distorted octahedral coordination at the Re atom. The central $\mathrm{CoReP}_{2}$ ring is nearly planar; the maximum deviation from the best plane is $0.02 \AA$ with a dihedral angle of $2.2^{\circ}$. With respect to the different metal atomic radii, the ring may be regarded as regular. It shows two equal $M-\mathrm{P}$ bond lengths for Co [2.111 (1) and 2.116 (1) $\AA$ ] as well as for $\operatorname{Re}[2.541$ (1) and 2.544 (1) $\AA$ ] . The enclosed ring angles at both $P$ atoms are acute [72.8 (1) and $\left.72.9(1)^{\circ}\right]$ and the $\mathrm{P}-M-\mathrm{P}$ angles reflect the distorted coordination polyhedron of each metal atom
The title should be concise yet descriptive. It must include the chemical name or the formula. Because all papers in Acta $C$ describe structures, phrases such as 'Structure of' may be omitted.

The authors' names should, where possible, include the full first name.
The Abstract should contain the full IUPAC chemical name and formula (if it is not present in the title), and basic data which identify the type of chemistry and structure studied. Any special geometry should be highlighted.

A chemical diagram is mandatory for molecular structure studies. This diagram will also be used for the Contents section of the journal ( $a$ synopsis will appear in the Contents listings for non-molecular structures).

Only the novel aspects of the structural geometry should be emphasized. These should be related to studies of similar structures and to studies of the compound using other techniques (NMR, IR, etc.). Any unusual geometry should be compared with the standard data listed in $\S 9$ of International Tables for Crystallography, Volume $C$. 


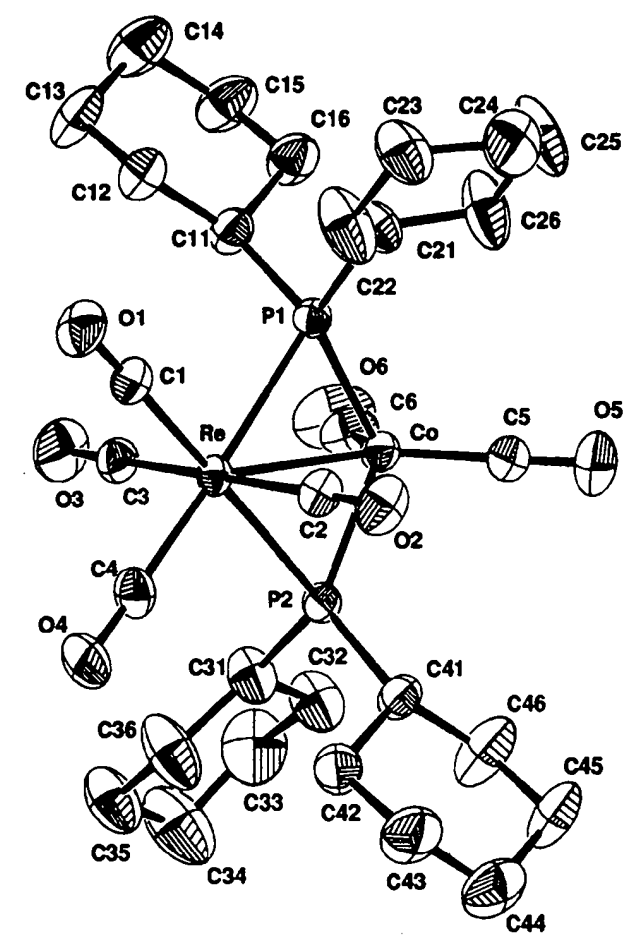

Fig. 1. Molecular structure showing $30 \%$ probability displacement ellipsoids. $\mathrm{H}$ atoms are omitted for clarity.

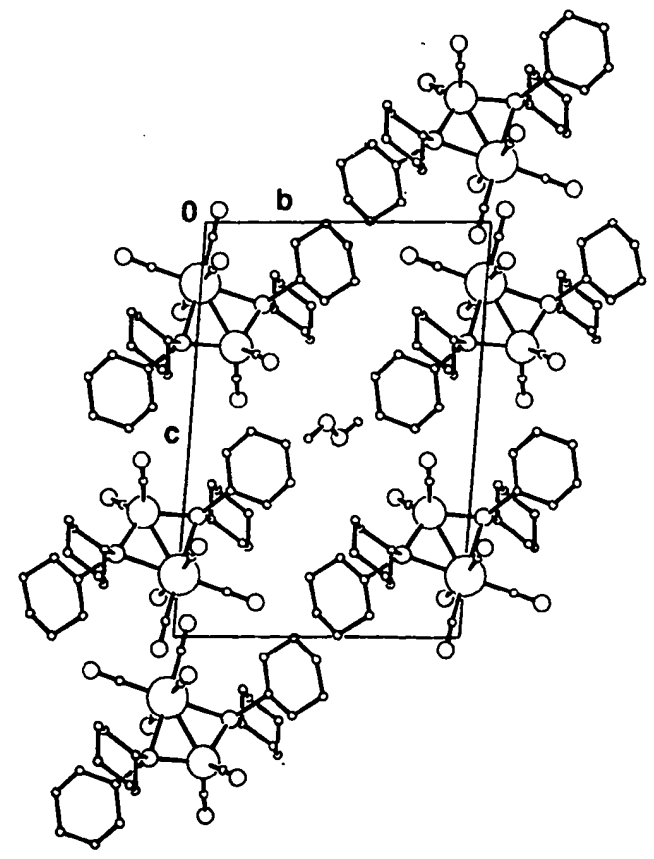

Fig. 2. Packing diagram viewed down the $a$ axis. Note the solvent molecule in the centre of the cell.
A good molecular diagram is essential for $a$ structural paper and it must be oriented to show the most important geometry. The atom labels must be readable when the diagram is reduced to one-column width $(80 \mathrm{~mm})$. Labels should clearly identify the atom sites and must match those listed in the table of atom coordinates and $U$ values. Ellipsoid plots are preferred to line, ball-and-stick or CPK plots because they aid visualization of the atomic displacements and hence provide disorder and thermal-motion information. Unrefined $\mathrm{H}$-atom sites may be omitted, or shown as balls. Distance and angle values should not be placed on the diagram.

A cell plot is useful if hydrogen bonding, solvent molecules or close van der Waals contacts need to be shown. This diagram can be stylized to emphasize packing information. The orientation of the cell must be displayed with respect to a set of right-handed axes. Hydrogen bonds are normally shown as dashed lines with the donor and acceptor sites suitably labelled. 
[121.3 (1) and $92.9(1)^{\circ}$ for $\mathrm{Co}$ and $\mathrm{Re}$, respectively]. The most interesting structural feature is the Co--Re single bond which meets the requirement of 18 valence electrons for each metal atom and has a length of 2.786 (1) $\AA$. We have established, by use of the Cambridge Structural Database (Allen et al., 1979), that the only other cobalt-rhenium cluster reported so far is $\left[\mathrm{Co}_{2} \operatorname{Re}\left(\mu_{3}\right.\right.$ $\left.\mathrm{CC}_{6} \mathrm{H}_{4} \mathrm{Me}_{4}\right)(\mathrm{CO})_{10}$ ] (Jeffery, Lewis, Lewis \& Stone, 1985), with Co- Re bond lengths of $2.686(1)$ and 2.720 (1) $\AA$. This triangular cluster has distinctly different bonding and bridging patterns, so direct comparison of the heteronuclear bond lengths of both compounds is not possible. However ...

\section{Experimental}

Synthesis was carried out by reaction of $\operatorname{Re}_{2}(\mathrm{CO})_{10}, \mathrm{Co}_{2}(\mathrm{CO})_{8}$ and $\mathrm{HP}\left(\mathrm{C}_{6} \mathrm{H}_{11}\right)_{2}$ (molar ratio $1: 1: 2$ ) in xylene solution for $10 \mathrm{~h}$ at $423 \mathrm{~K}$ in a glass tube. Recrystallization was from $\mathrm{MeOH}$.

\section{Crystal data}

$\left[\mathrm{CoRe}\left(\mathrm{C}_{12} \mathrm{H}_{22} \mathrm{P}\right)_{2}(\mathrm{CO})_{6}\right]$.$0.5 \mathrm{CH}_{3} \mathrm{OH}$

$M_{r}=823.7$

Triclinic

$$
P \overline{1}
$$$$
a=10.452(3) \AA
$$$$
b=11.664(4) \AA
$$$$
c=15.641(4) \AA
$$$$
\alpha=94.37(2)^{\circ}
$$$$
\beta=89.75(2)^{\circ}
$$$$
\gamma=111.87(2)^{\circ}
$$$$
V=1763.8(8) \AA^{3}
$$$$
Z=2
$$$$
D_{x}=1.551 \mathrm{Mg} \mathrm{m}^{-3}
$$$$
D_{m} \text { not measured }
$$

Data collection

Siemens $R 3 m / V$ diffractometer

$\omega-2 \theta$ scans

Absorption correction: empirical via $\psi$ scans $T_{\min }=0.690, T_{\max }=$ 0.894

15189 measured reflections

8161 independent reflections 6813 observed reflections

$$
[F>4 \sigma(F)]
$$

\begin{tabular}{|c|c|}
\hline Refinement on $F$ & $w=1 /\left[\sigma^{2}(F)+0.0001 F^{2}\right]$ \\
\hline $\begin{array}{l}R=0.038 \\
w R=0.034\end{array}$ & $\begin{array}{l}(\Delta / \sigma)_{\max }=0.001 \\
\Delta \rho_{\max }=0.95 \mathrm{e}^{-3}\end{array}$ \\
\hline$S=1.583$ & $\Delta p_{\min }=-0.80 \mathrm{e} \AA^{-3}$ \\
\hline 6813 reflections & Extinction correction: none \\
\hline 379 parameters & Atomic scattering factors \\
\hline $\begin{array}{l}\text { H-atom parameters not } \\
\text { refined }\end{array}$ & $\begin{array}{l}\text { from SHELXTL-Plus } \\
\text { (Sheldrick, 1990) }\end{array}$ \\
\hline
\end{tabular}

Refinement

$R_{\text {int }}=0.022$

$h=-13 \rightarrow 13$

$k=-15 \rightarrow 15$

$l=-21 \rightarrow 21$

4 standard reflections monitored every 400 reflections intensity decay: none
If there is more than one structure, each experimental data set should be identified with a sequence code [e.g. (1), (2), ... or (I), (II), ...].

A brief summary of the chemical and crystal preparation should be given in sufficient detail to reproduce these aspects of the experiment.

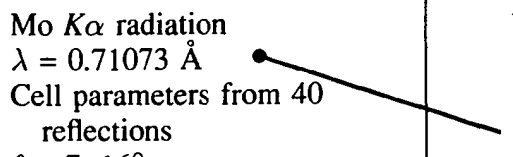

$\theta=7-16^{\circ}$ $\mu=4.07 \mathrm{~mm}^{-1}$ $T=293 \mathrm{~K}$ Prism $0.50 \times 0.34 \times 0.28 \mathrm{~mm}$ Red

The wavelength $\lambda$ refers to the radiation used

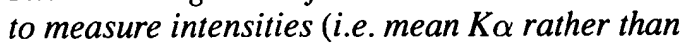
$\left.K \alpha_{1}\right)$.

The absorption coefficient $\mu$ is in $\mathrm{mm}^{-1}$ not $\mathrm{cm}^{-1}$.

The calculated density, $D_{x}$, must be supplied; $D_{m}$ should be measured if at all possible and details of the method given.

$\theta_{\max }$ refers to the diffraction angle $\theta$ not $2 \theta$. The $R$ value is always calculated on $F$, whereas $w R$ is calculated with the coefficient used in the least-squares refinement $\left(F, F^{2}\right.$ or $\left.I\right)$. 
